\title{
Percepción de la Satisfacción Laboral del Personal de Enfermería del Hospital General de Ticomán
}

\author{
Eulalia García Salazar (Licenciada en Enfermería) \\ Gustavo Argenis Hernández-Segura (Maestro en Investigación \\ Médica) \\ María Carlota García-Gutiérrez (Doctora en Ciencias) \\ Ruth Magdalena Gallegos-Torres (Doctora en Ciencias de la \\ Salud) \\ Universidad Autónoma de Querétaro, Querétaro, México
}

Doi:10.19044/esj.2020.v16n21p347 URL:http://dx.doi.org/10.19044/esj.2020.v16n21p347

\section{Resumen}

Introducción: Es importante investigar las organizaciones para conocer su funcionamiento y cómo este impacta en la insatisfacción de los trabajadores. Objetivo. Establecer la percepción de la satisfacción laboral en el personal de enfermería Hospital General de Ticomán. Metodología: Estudio transversal, descriptivo; instrumento Font-Roja, 24 ítems en una escala tipo Likert con 9 factores. Tamaño de muestra de 133 enfermeras, con la fórmula para poblaciones finitas, donde $\mathrm{p}=0.5$ y hay un nivel de confianza del $95 \%$. Tipo de muestreo por conveniencia; se tomaron a las enfermeras de los turnos, matutino, vespertino, velada A, B, Mixta y turno especial y que tuvieran disponibilidad para la participación. Proyecto fue evaluado por un Comité de Investigación y Bioética. Se respetaron los principios éticos para la investigación en seres humanos. Resultados: Personal entre 18 y 60 años, $70 \%$ mujer. $46 \%$ tenía estudios de nivel técnico en enfermería. En cuanto a la antigüedad, $26 \%$ se encuentran por debajo de los 5 años. $51 \%$ se encuentra muy satisfecho en el trabajo, $35 \%$ están de acuerdo en que el reconocimiento en el trabajo es muy reconfortable, $42 \%$ están de acuerdo que con frecuencia sienten no tener recursos suficientes. Conclusiones: La satisfacción laboral es un fenómeno presente en cualquier institución o área de trabajo, independiente del ramo que se aborde. Si hay atención a personas es más relevante porque influirá en la manera en que se atiende a los usuarios. En el área de la salud su relevancia radica que tiene implicaciones hacia la educación de la población.

Palabras clave: Enfermeras, Factores intrínsecos, Satisfacción labora 


\title{
Perception of Job Satisfaction of Nursing Staff at Ticomán General Hospital
}

\author{
Eulalia Garcia Salazar (Licenciada en Enfermería) \\ Gustavo Argenis Hernández-Segura (Maestro en Investigación \\ Médica) \\ María Carlota Garcia-Gutiérrez (Doctora en Ciencias) \\ Ruth Magdalena Gallegos-Torres (Doctora en Ciencias de la \\ Salud) \\ Universidad Autónoma de Querétaro, Querétaro, México
}

\begin{abstract}
Introduction: It is important to investigate organizations to better understand how they work and the significant impact they have on the dissatisfaction of workers. Objective: To establish the perception of job satisfaction in the nursing staff of the General Hospital at Ticomán. Methodology: Cross-sectional and descriptive study, using Font-Roja instrument, of 24 items was represented on a 9-point Likert scale. The sample size was 133 nurses, and this is determined by the formula for finite populations, where $\mathrm{p}=0.5$ with a $95 \%$ confidence level. The type of sampling used was convenience sampling because the nurses who worked during the investigation period of the shifts (morning, evening, evening A, B, Mixed and special shift) were available for participation. The project was evaluated by a Research and Bioethics Committee. Ethical principles for research in human beings were respected. Results: 70\% female personnel are between 18 and 60 years old. $46 \%$ had technical level studies in nursing. As for seniority, $26 \%$ are below 5 years. $51 \%$ are very satisfied at work, $35 \%$ agree that recognition at work is very comfortable, and $42 \%$ agree that they often feel they do not have enough resources. Conclusion: Job satisfaction is a phenomenon present in any institution or work area, regardless of the branch being addressed. If customer service is available, it becomes more relevant because it will influence the way users are served. In the area of health, its relevance lies on its implications for the education of the population.
\end{abstract}

Keywords: Nurses, Intrinsic factors, Job satisfaction 


\section{Introducción}

Las organizaciones, se han constituido ahora más que nunca, en las instituciones dominantes de la sociedad, independientemente del área en la que trabajen, ya que hay países cláramente industrializados o cuya dinámica económica está dada por múltiples organizaciones. A través de ellas, las personas pueden alcanzar metas que individualmente no podrían lograr; una de ellas puede ser el estar involucrado en la producción y distribución de la mayoría de los bienes y servicios que la sociedad requiere para poder satisfacer sus necesidades (Troya, Padilla, Camacho, \& Benavides Laura, 2020). De allí deriva la importancia del estudio de las organizaciones, cómo están estructuradas, cuál es la dinámica al interior de sus procesos y en su funcionamiento y cómo todo esto influye en la conducta de los individuos (Piqueras Gómez, Rodríguez Morejón, \& Rueda Sabater, 2008).

Específicamente hablando, las instituciones de salud juegan un papel muy importante en la socidad, más en los actuales tiempos de tanta transformación social, la presencia de nuevas patologías y fenómenos, entre otros. Los hospitales son de los lugares que requieren una organización muy detallada en sus procesos por lo que, por ejemplo, el ciclo de trabajo del personal de enfermería necesita estar bastante definido. Las instituciones hospitalarias son espacios en donde interactúa personal del área de la salud, de áreas sociales, humanidades, administrativas, nutriólogos, personal de limpieza, seguridad, y muchos otros, además de las personas enfermas y sanas; de la misma manera existen con certeza, emociones complejas de cada una de estas, por lo que es un área con una diversidad de factores que pudieran influenciar la calidad de la atención (Parra \& Paravic, 2002).

Al respecto, los trabajadores adoptan ciertas actitudes ante el actuar diario que pudieran no ser siempre favorables para el paciente. Esta acción puede provocar un "contagio" con el resto del mismo gremio y generar un clima laboral desfavorable. Es por ello que en todas las organizaciones y especialmente en las de entorno sanitario, es cada vez más necesario concientizar a los profesionales hacia el trabajo de calidad, por lo que día con día se deben de implementar programas que cuenten con objetivos actualizados, que contengan estrategias novedosas y prácticas que contribuyan al desarrollo del recurso humano (Piqueras Gómez et al., 2008).

Relacionado con lo anterior, en la década de los 30, se iniciaron los estudios sistemáticos sobre la satisfacción laboral y los factores que podrían afectarla. Hoppock en 1935 publicó la primera investigación en donde hacía un análisis profundo de la satisfacción laboral. A través de sus resultados llegó a la conclusión de que "existen múltiples factores que podrían ejercer influencia sobre la satisfacción laboral, dentro de los cuales hizo mención a la fatiga, monotonía, condiciones de trabajo y supervisión" (Parra \& Paravic, 2002). 
La satisfacción laboral, siguiendo la definición dada por Locke en 1976, se entiende que parte de la apreciación, es decir, de la percepción personal que cada individuo hace de su trabajo, lo que le lleva a valorar o apreciar el área en donde está o de la organización en su totalidad, siendo estos valores congruentes o de ayuda para satisfacer sus necesidades básicas, las cuales pueden ser de tipo físico o psicológico, afectivo y emocional, dando a la persona una perspectiva de un antes y después, es decir, un punto de comparación (Al-Shammari, 1992; Germán Bes, Hueso Navarro, \& Huércanos Esparza, 2011).

A nivel teórico, como lo señala Herzberg, se ha comprobado que la satisfacción laboral se encuentra influenciada por dos factores, el intrínseco y el extrínseco. El primero, también llamado "motivación”, incluye el desarrollo personal, la responsabilidad, el reconocimiento, el propio trabajo, las fortalezas y las metas. El segundo, igualmente denominado "higiene", engloba la política de la compañía, el salario, las relaciones interpersonales con el supervisor y con el resto del equipo profesional, condiciones laborales, la vida personal, el estatus y la seguridad (Germán Bes et al., 2011).

Sin embargo, las dimensiones que con mayor frecuencia han sido descritas como influyentes en una organización son las condiciones de trabajo, la formación académica, promoción y desarrollo profesional, reconocimiento, retribución, relación jerárquica, participación, organización y gestión del cambio, relación con los compañeros, comunicación interna, conocimiento e identificación de objetivos y la percepción de la dirección (Cardona Echeverri \& Zambrano Cruz, 2014).

Al respecto, el compromiso laboral va más allá de cumplir con una obligación, implica hacer uso de las capacidades personales y profesionales de cada individuo para sacar adelante todo aquello que se le ha confiado. En este sentido, una persona comprometida se reconoce como aquella que cumple con sus obligaciones, haciendo un poco más de lo esperado al grado de sorprender, porque vive, piensa y sueña con sacar adelante a su familia, su trabajo, su estudio y todo aquello que es importante en su vida tanto de forma laboral como profesional (Piqueras Gómez et al., 2008).

El impacto de estas dimensiones va a depender de las características propias de cada organización, es decir, de lo que cada persona desee y logre hacer con base en sus habilidades y capacidades, por tanto, es necesario estudiar qué dimensiones con mayor impacto están influyendo en la valoración del grado de satisfacción de los trabajadores, para poder diseñar planes de gestión eficaces y dirigidos a las necesidades de los profesionales que las integran (Parra \& Paravic, 2002).

Una de las organizaciones primordiales de la sociedad son las del sector salud, quienes tienen la consigna de proporcionar cuidados a la población, sana o enferma, a través de prácticas de calidad, dadas por un 
equipo multidisciplinario, donde la enfermera es una pieza clave, brindando una atención de calidad y calidez al paciente, familia y comunidad (Ceballos Vásquez, 2010).

Las condiciones laborales y la insatisfacción por parte del personal en el sector salud no se limita solo a México. Éste es un fenómeno mundial que se estudió en 2011 en España (Germán Bes et al., 2011), y en 2015 en Murcia (Carrillo-García et al., 2015).

En un estudio llevado a cabo en el 2017 en Santiago de Chile, se encontró que los profesionales de Enfermería sufren una gran frustración profesional, con altas puntuaciones de estados depresivos. Se demostró que las enfermeras que brindan sus cuidados en servicios de hospitalización presentan índices mayores de depresión, estrés y frustración profesional y por ende menores niveles de satisfacción laboral (Silva, 2017).

Otro estudio, realizado en un hospital de gineco-obstetricia del Instituto Mexicano del Seguro Social en el Distrito Federal, encontró que los trabajadores presentaron altos niveles de insatisfacción con respecto a la remuneración económica, salud y condiciones laborales. Al respecto, Briseño detectó bajo salario y pocas posibilidades de ascenso, condiciones que producen mayor insatisfacción entre el personal de enfermería (Ponce-Gómez, Ponce-Gómez, \& Lugo-Chávez, 2009).

Si bien la satisfacción laboral y el trabajo eficiente poseen una alta relación, dado que es alta la probabilidad de que una persona satisfecha trabaje eficientemente en los procesos, se fomente el trabajo en equipo, aumente la calidad de la atención de enfermería y el rendimiento de su productividad, también se conoce que lo anterior no es una garantía, dada la múltiple variedad de factores que pueden influir. Lo que sí es evidente es que la satisfacción de los usuarios garantiza el cumplimiento de los indicadores de calidad y las normas de atención institucionales (Figueiredo Manzo, Teodoro Couto Ribeiro, Menezes Brito, \& Alves, 2012).

Al respecto, Margarita Hernández Zavala, en un estudio realizado en cuatro instituciones públicas de salud del tercer nivel de atención, siendo estos el Instituto Nacional de Pediatría, Instituto Nacional de Neurocirugía, Instituto Nacional de Enfermedades Respiratorias Ismael Cosio Villegas y el Centro de Investigación Materno Infantil grupo de Estudio al Nacimiento, encuestó a 594 profesionales de enfermería y encontró que la satisfacción laboral en estas instituciones tiene una calificación de nivel medio a alto; así mismo reconoció que es relevante la identificación de factores de insatisfacción así como de satisfacción, de manera tal que con ellas se puedan fundamentar mejoras e incidir en la calidad de los cuidados (Hernández Zavala et al., 2012).

En otro estudio realizado por Molina en un hospital médico-quirúrgico donde seleccionaron aleatoriamente a 75 profesionales de un total de 510 enfermeras, se encontró que la satisfacción laboral del profesional de 
enfermería es un concepto importante que puede incidir en los motivos relacionados con la identidad laboral y pertenencia en el lugar de desempeño y por tanto, en la estabilidad de las plantillas en el hospital y es, a su vez, un concepto clave en la mejora de la calidad de los cuidados que se otorgan a los pacientes. Es relevante destacar como factores estructurales, cómo el bloque o unidad en la que se trabaja pueden influir o modular el grado de satisfacción (Hernández Zavala et al., 2012; Jaramillo Antillón, 1998; Molina Linde, Avalos Martínez, Valderrama Orbegozo, \& Uribe Rodríguez, 2009).

En una investigación llevada en dos hospitales de Cuba, relacionada noc la motivación y el liderazgo, fue evidente que en la motivación infuyen las relaciones que el personal tiene con las personas que les rodean, así como el sentido de pertenencia, es decir, donde los logros indican que se cumplen las funciones de manera cabal además que apoyan al cumplimiento de las metas planteadas (Sánchez Jacas, Brea López, De La Cruz Castro, \& Matos Fernández, 2017).

En un estudio de Soto llevado a cabo en Chile, en un hospital público, trabajado con 35 enfermeras, reflejó que estas tenían, en los datos generales, un nivel medio de satisfacción laboral. Las dimensiones de puntajes altos fueron adecuación al trabajo, relación laboral y satisfacción en el trabajo. Los puntajes más bajos fueron "distensión en el trabajo, presión y control en el trabajo" (Soto, Barrios, \& Molina, 2017).

Así mismo, en un estudio realizado a 280 enfermeros en Bogotá, Colombia, se encontró que favorecen la labor profesional la satisfacción por el trabajo y la competencia profesional; en este sentido la afectan la monotonía y el grado en que un individo percibe su carga laboral. Con respecto a la monotonía, se encontró que esta es más evidente en ámbitos no hospitalarios (Herrera-Amaya \& Manrique-Abril, 2019).

Dentro de la definición de calidad de la atención, se consideran aspectos del bienestar de los trabajadores, vinculados al aspecto físico, mental y social, que a su vez impactan en estos mismos rubros de los que reciben la atención. En la actualidad se fomenta el desarrollo de investigaciones en las instituciones prestadoras de servicios en salud, enfocadas a la calidad de la atención y los servicios ofrecidos, evaluando la satisfacción del usuario, sin embargo, en pocas ocasiones se tienen en cuenta las condiciones del trabajador y su satisfacción laboral, lo cual representa un factor importante para lograr los objetivos, metas y estándares de una institución (Hernández Zavala et al., 2012; Molina Linde et al., 2009).

En este sentido, el aumento de la satisfacción laboral del profesional de enfermería optimiza la relación con el paciente, familia, comunidad y el mismo personal de salud incrementando la calidad de los cuidados prestados (Núñez González, Estévez Guerra, Hernández Marrero, \& Marrero Medina, 2007). 
El nivel de satisfacción de las personas con su trabajo en una organización, impacta en la reputación de la misma, tanto a nivel interno como externo y las diferencias entre unidades organizacionales en satisfacción laboral son vistas como síntomas preocupantes de potenciales deficiencias, por lo cual, todo ello convierte a la satisfacción laboral en un aspecto prioritario de evaluación y mejora en cualquier organización, con mayor motivo en el campo administrativo, donde la labor que realizan tiene como materia prima a las personas en formación y como objetivo guiar eficazmente su desarrollo hasta lograr que estén plena e íntegramente identificados con su labor. En este sentido, esta investigación se encaminó a determinar la percepción de la satisfacción laboral del personal de enfermería del Hospital General de Ticomán, de la Ciudad de México.

\section{Metodología}

Se llevó a cabo un estudio transversal, descriptivo. El universo estuvo conformado por enfermeras que trabajan en el Hospital General de Ticomán teniendo un estimado de 264. Se tomaron a las enfermeras y enfermeros que laboraran en el periodo de la investigación de los turnos matutino, vespertino, velada A, B, mixta y turno especial (fines de semana y días festivos) de los servicios de Consulta Externa, Urgencias, Cirugía General, Medicina Interna, Unidad de Terapia Intensiva Adultos, Quirófano, CEyE, Ginecología y Unidad de Terapia Intensiva Neonatales del Hospital General.

El tamaño de la muestra fue de 133 enfermeras, esto determinado mediante la fórmula para poblaciones finitas, donde $\mathrm{p}=0.5 \mathrm{y}$ un nivel de confianza del $95 \%$. El tipo de muestreo fue por conveniencia, ya que por las condiciones de la organización, se proporcionó la herramienta de recolección de datos a aquellos que tenían disponibilidad de tiempo y deseaban participar en el estudio, previa entrega del respectivo consentimiento informado.

Se trabajó con el cuestionario Font-Roja de satisfacción laboral, el cual también se ha usado en otros estudios (Herrera-Amaya \& Manrique-Abril, 2019; Soto et al., 2017); consta de 24 ítems representados en una escala tipo Likert (evaluados entre 1=muy malo y 5=muy bueno), agrupados en 9 factores que permiten explorar diferentes dimensiones que intervienen en la satisfacción laboral: $a$ ) satisfacción por el trabajo, $b$ ) tensión relacionada con el trabajo, $c$ ) competencia profesional, $d$ ) presión en el trabajo, e) promoción profesional, f) relación interpersonal con los superiores, g) relación interpersonal con los compañeros, $h$ ) características extrínsecas de estatus y $i$ ) monotonía laboral (Ponce-Gómez et al., 2009).

Este instrumento es una adaptación del Tecumeh Community Meath Study, realizada por Aranaz en el año 1988. Al documento utilizado se le agregaron preguntas sociodemográficas como: edad, sexo, estado civil, grado académico, antigüedad laboral e interés de estudiar, esto sin modificar la 
sección que estudia la satisfacción laboral. La información se analizó mediante el uso de estadística deskriptiva obteniendo frecuencias.

El proyecto fue sometido a evaluación por un Comité de Investigación y Bioética. Se respetaron los principios éticos para la investigación en seres humanos.

\section{Resultados}

Se presentan los datos de 231 enfermeras. La edad de los participantes estuvo entre los 18 y 60 años de edad. El 19.0\% (44) tuvieron entre 36 y 41 años y 4\% (1) reportó 60 años. También se encontró que la mayoría son mujeres, representado con el 70\% (162) y el 30\% (69) son hombres. En cuanto al estado civil de los participantes, la mayoría son casados, con un $41 \%$ (94). El grado académico predominante fue técnico de enfermería con un 46\% (106) y la minoría es de $3 \%$ con posgrado (8). La antiguiedad laboral se aprecia en la Figura 1.

Figura 1. Antigüedad laboral de los participantes en años

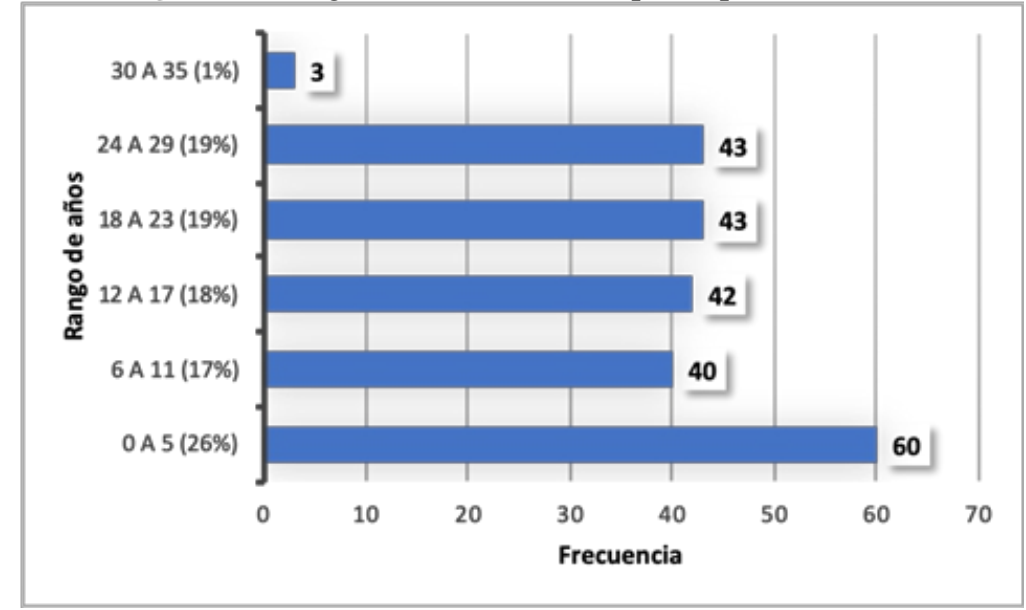

Fuente: instrumento aplicado

Los aspectos laborales, se reflejan en la Figura 2.

Se encontró que con respecto al clima laboral, el 40\% (92) está de acuerdo en que muy pocas veces se siente que el trabajo perturba su salud, el $51 \%$ (119) refiere estar en desacuerdo en que le falta tiempo para realizar el trabajo y que no se dan abasto con lo que se tiene que hacer, sólo el 6\% (14) está muy en desacuerdo sobre contar con el abasto suficiente para realizar bien su trabajo, sin embargo el $42 \%$ (98) con frecuencia indica que la competitividad, o el estar a la altura de los demás, en su puesto de trabajo, le causa estrés o tensión.

En las relaciones laborales, el 21\% (49) de los participantes refieren estar de acuerdo en que estas son cordiales con sus jefas/es, y con los compañeros el 49\% (113). En cuanto a la insatisfacción laboral, el 85\% (131) 
está en desacuerdo en tener muy poco interés en las cosas que realizan en su trabajo y solo el 2\% (5) tiene la sensación de que lo que hace no vale la pena.

Con respecto al reconocimiento, el $51 \%$ (118) del personal encuestado está de acuerdo en sentirse muy satisfechos en su trabajo, solo el 8\% (19) señala tener pocas oportunidades para aprender a hacer cosas nuevas; $14 \%$ (33) refiere sentirse que el reconocimiento que obtiene en el trabajo es muy reconfortante.

En cuanto a la adecuación al puesto, el 5\% (11) de los encuestados indican tener poca libertad e independencia para organizar el trabajo, el 53\% (121) refiere estar de acuerdo en estar convencido que el puesto de trabajo que ocupa le corresponde por su capacidad de preparación.

Con respecto a la promoción, el $51 \%$ (119) está de acuerdo en tener capacidad de promoción, solo el 4\% (9) está muy en desacuerdo de estar seguro de conocer lo que se espera de su trabajo, por parte de sus jefes/as.

Figura 2. Frecuencia de las respuestas vinculadas a los aspectos laborales en los trabajadores.

\begin{tabular}{|c|c|c|c|c|c|}
\hline PREGUNTAS & $\begin{array}{l}\text { Muy de } \\
\text { acuerdo }\end{array}$ & De acuerdo & $\begin{array}{c}\text { Ni de } \\
\text { acuerdo ni } \\
\text { en } \\
\text { desacuerdo }\end{array}$ & $\begin{array}{c}\text { En } \\
\text { desacuerdo }\end{array}$ & $\begin{array}{c}\text { Muy en } \\
\text { desacuerdo }\end{array}$ \\
\hline $\begin{array}{l}\text { 1. Mi actual trabajo en el } \\
\text { Hospital es el mismo todos } \\
\text { los días, no varía nunca. }\end{array}$ & $14(6 \%)$ & $43(19 \%)$ & $39(17 \%)$ & $90(39 \%)$ & $45(6 \%)$ \\
\hline $\begin{array}{l}\text { 2. Al final de la jornada de } \\
\text { un día de trabajo corriente y } \\
\text { normal, me suelo encontrar } \\
\text { muy cansado(a). }\end{array}$ & $14(6 \%)$ & $59(26 \%)$ & $62(27 \%)$ & $77(33 \%)$ & $19(8 \%)$ \\
\hline $\begin{array}{l}\text { 3. Con bastante frecuencia } \\
\text { me he sorprendido fuera del } \\
\text { Hospital pensando en } \\
\text { cuestiones relacionadas con } \\
\text { mi trabajo. }\end{array}$ & $11(5 \%)$ & $55(24 \%)$ & $43(18 \%)$ & $99(43 \%)$ & $23(10 \%)$ \\
\hline $\begin{array}{l}\text { 4. Muy pocas veces me he } \\
\text { obligado a emplear "a tope" } \\
\text { toda mi energía y capacidad } \\
\text { para realizar mi trabajo. }\end{array}$ & $14(6 \%)$ & $46(20 \%)$ & $43(18 \%)$ & $101(44 \%)$ & $27(12 \%)$ \\
\hline $\begin{array}{l}\text { 5. Muy pocas veces mi } \\
\text { trabajo en el Hospital } \\
\text { perturba mi estado de } \\
\text { ánimo, o a mi salud o a mis } \\
\text { horas de sueño. }\end{array}$ & $18(8 \%)$ & $42(18 \%)$ & $46(20 \%)$ & $92(40 \%)$ & $33(14 \%)$ \\
\hline $\begin{array}{l}\text { 6. En mi trabajo me } \\
\text { encuentro muy satisfecho. }\end{array}$ & $87(38 \%)$ & $118(51 \%)$ & $16(7 \%)$ & $4(2 \%)$ & $6(2 \%)$ \\
\hline $\begin{array}{l}\text { 7. Para organizar el trabajo } \\
\text { que realizo, según mi puesto } \\
\text { concreto o categoría } \\
\text { profesional, tengo poca }\end{array}$ & $11(5 \%)$ & $26(11 \%)$ & $48(21 \%)$ & $111(48 \%)$ & $35(15 \%)$ \\
\hline
\end{tabular}




\begin{tabular}{|c|c|c|c|c|c|}
\hline $\begin{array}{l}\text { independencia (es decir, } \\
\text { poca libertad). }\end{array}$ & & & & & \\
\hline $\begin{array}{l}\text { 8. Tengo pocas } \\
\text { oportunidades para aprender } \\
\text { a hacer cosas nuevas. }\end{array}$ & $19(8 \%)$ & $28(12 \%)$ & $31(14 \%)$ & $104(45 \%)$ & $49(21 \%)$ \\
\hline $\begin{array}{l}\text { 9. Tengo muy poco interés } \\
\text { por las cosas que realizo en } \\
\text { mi trabajo. }\end{array}$ & $2(1 \%)$ & $5(3 \%)$ & $9(6 \%)$ & $131(85 \%)$ & $84(5 \%)$ \\
\hline $\begin{array}{l}\text { 10. Tengo la sensación de } \\
\text { que lo que estoy haciendo } \\
\text { no vale la pena }\end{array}$ & $5(2 \%)$ & $6(3 \%)$ & $8(3 \%)$ & $110(48 \%)$ & $102(44 \%)$ \\
\hline $\begin{array}{l}\text { 11. Generalmente el } \\
\text { reconocimiento que obtengo } \\
\text { en mi trabajo es muy } \\
\text { reconfortable. }\end{array}$ & $33(14 \%)$ & $80(35 \%)$ & $46(20 \%)$ & $58(25 \%)$ & $14(6 \%)$ \\
\hline $\begin{array}{l}\text { 12. La relación con mi } \\
\text { jefe/a, jefes/as es muy } \\
\text { cordial. }\end{array}$ & $49(21 \%)$ & $102(44 \%)$ & $53(23 \%)$ & $21(9 \%)$ & $6(3 \%)$ \\
\hline $\begin{array}{l}\text { 13. La relación con mis } \\
\text { compañeros/as son muy } \\
\text { cordiales. }\end{array}$ & $37(16 \%)$ & $113(49 \%)$ & $60(26 \%)$ & $18(8 \%)$ & $3(1 \%)$ \\
\hline $\begin{array}{l}\text { 14. El sueldo que percibo es } \\
\text { muy adecuado. }\end{array}$ & $20(9 \%)$ & $86(37 \%)$ & $57(25 \%)$ & $55(24 \%)$ & $13(5 \%)$ \\
\hline $\begin{array}{l}\text { 15. Estoy convencido que el } \\
\text { puesto de trabajo que ocupo } \\
\text { es el que me corresponde } \\
\text { por capacidad y } \\
\text { preparación. }\end{array}$ & $34(15 \%)$ & $121(53 \%)$ & $31(13 \%)$ & $31(13 \%)$ & $14(6 \%)$ \\
\hline $\begin{array}{l}\text { 16. Tengo muchas } \\
\text { capacidades de promoción } \\
\text { profesional. }\end{array}$ & $44(19 \%)$ & $119(51 \%)$ & $43(19 \%)$ & $18(8 \%)$ & $7(3 \%)$ \\
\hline $\begin{array}{l}\text { 17. Con frecuencia tengo la } \\
\text { sensación de que me falta } \\
\text { tiempo para realizar mi } \\
\text { trabajo. }\end{array}$ & $13(6 \%)$ & $44(19 \%)$ & $37(16 \%)$ & $119(51 \%)$ & $18(8 \%)$ \\
\hline $\begin{array}{l}\text { 18. Estoy seguro(a) de } \\
\text { conocer lo que se espera de } \\
\text { mi trabajo, por parte de mis } \\
\text { jefes/as. }\end{array}$ & $19(8 \%)$ & $113(49 \%)$ & $55(24 \%)$ & $35(15 \%)$ & $9(4 \%)$ \\
\hline $\begin{array}{l}\text { 19. Creo que mi trabajo es } \\
\text { excesivo, no doy abasto con } \\
\text { lo que hay que hacer. }\end{array}$ & $6(3 \%)$ & $38(16 \%)$ & $56(24 \%)$ & $102(44 \%)$ & $29(13 \%)$ \\
\hline $\begin{array}{l}\text { 20. Los problemas } \\
\text { personales de mis } \\
\text { compañeros/as de trabajo, } \\
\text { habitualmente me suelen } \\
\text { afectar. }\end{array}$ & $4(2 \%)$ & $16(7 \%)$ & $51(22 \%)$ & $105(45 \%)$ & $55(24 \%)$ \\
\hline $\begin{array}{l}\text { 21. Con frecuencia siento } \\
\text { que los recursos de la } \\
\text { institución no son }\end{array}$ & $20(9 \%)$ & $49(21 \%)$ & $23(10 \%)$ & $84(36 \%)$ & $55(24 \%)$ \\
\hline
\end{tabular}




\begin{tabular}{|l|l|l|l|l|l|}
\hline $\begin{array}{l}\text { suficientes para hacer bien } \\
\text { mi trabajo. }\end{array}$ & & & & & \\
\hline $\begin{array}{l}\text { 22. Con frecuencia siento } \\
\text { no tener recursos suficientes } \\
\text { para realizar mi trabajo tan } \\
\text { bien como sería deseable. }\end{array}$ & $48(21 \%)$ & $\mathbf{9 8 ~ ( 4 2 \% )}$ & $37(16 \%)$ & $34(15 \%)$ & $14(6 \%)$ \\
\hline $\begin{array}{l}\text { 23. Con frecuencia la } \\
\text { competitividad, o el estar a } \\
\text { la altura de los demás, en mi } \\
\text { puesto de trabajo, me causa } \\
\text { estrés o tensión. }\end{array}$ & $2(1 \%)$ & $18(8 \%)$ & $69(30 \%)$ & $\mathbf{8 8 ~ ( 3 8 \% )}$ & $54(23 \%)$ \\
\hline
\end{tabular}

Fuente: instrumento aplicado

Se encontró que el $51 \%$ (118) del personal encuestado se encuentra muy satisfecho en el trabajo, como lo muestra la Figura 3.

Figura 3. Referencia sobre la satisfacción en el trabajo, por parte del personal de enfermería

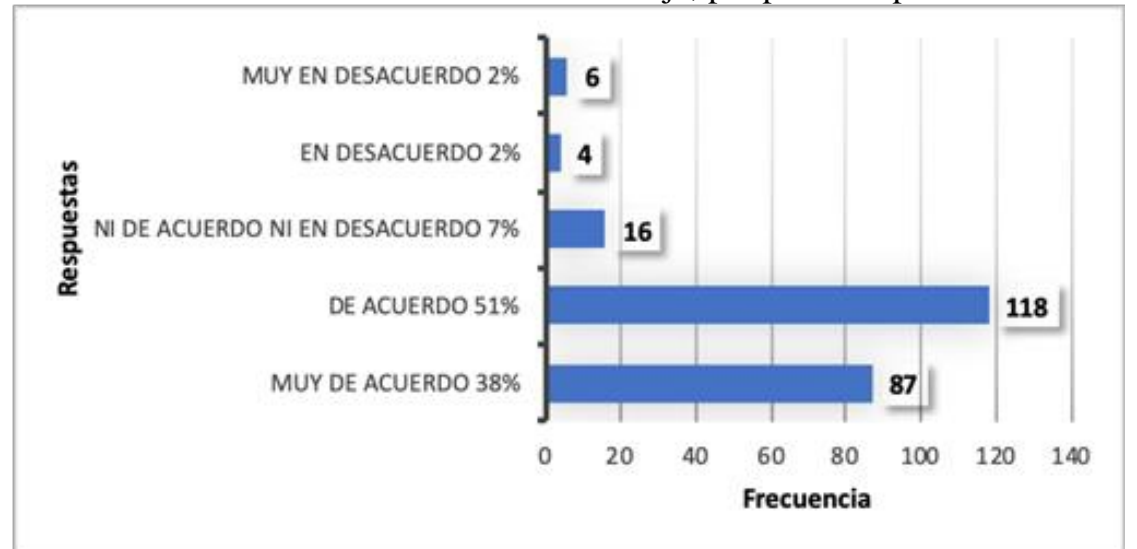

Fuente: instrumento aplicado

El 35\% (80) están de acuerdo en que generalmente el reconocimiento en el trabajo es muy reconfortable, tal como se observa en la Figura 4.

Figura 4. Reconocimiento percibido por el trabajo realizado

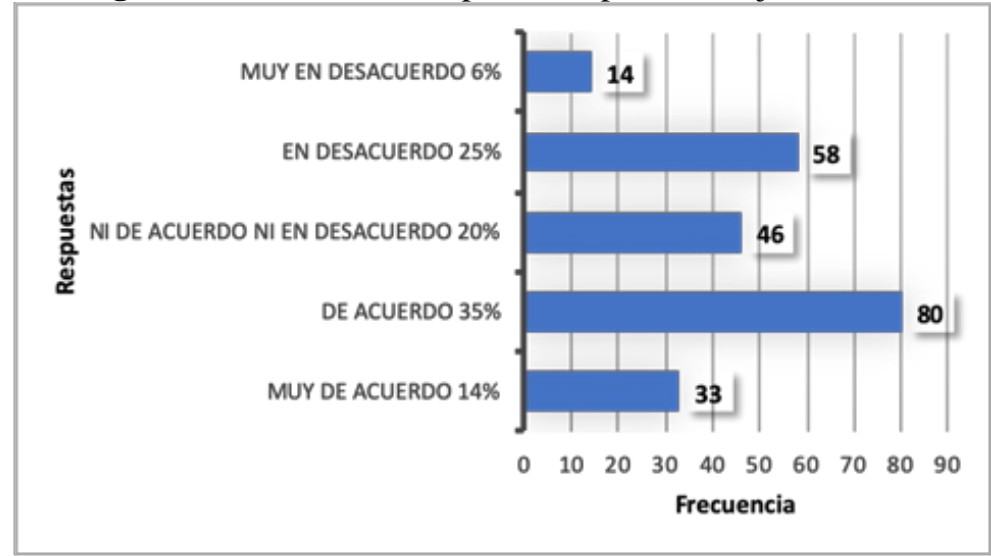

Fuente: instrumento aplicado 
El 42\% (98) de los participantes están de acuerdo que con frecuencia sienten no tener recursos suficientes para realizar su trabajo tan bien como desearían, como se muestra en la Figura 5.

Figura 5. Percepción sobre la posibilidad de no tener recursos para realizar el trabajo.

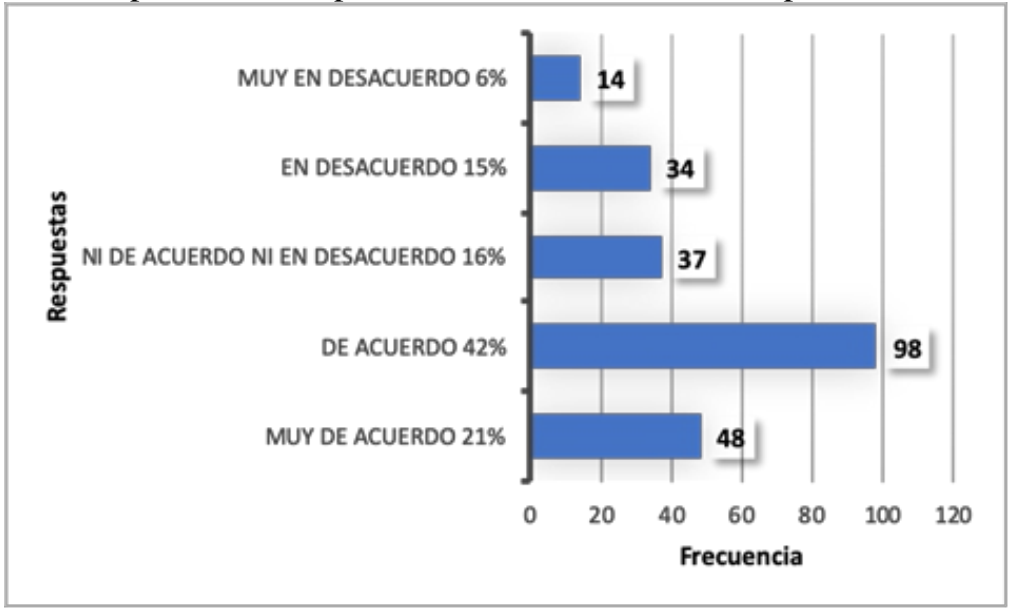

Fuente: instrumento aplicado

\section{Discusión y conclusión}

El tema respecto a la satisfacción laboral del personal de enfermería implica una enorme polémica, puesto que los aspectos a considerar son múltiples y de diversa índole; es por ello que una valoración de este tipo en todos los niveles de atención es relevante, sin embargo, en ocasiones se pudiera percibir que no hay interés en conocer el sentimiento, deseo, emoción e impresión afectiva de cada individuo como lo marca la teoría de Herzberg, quien indica que los factores intrínsecos y extrínsecos influyen directamente en la satisfacción laboral (Germán Bes et al., 2011).

$\mathrm{Al}$ respecto, diferentes teóricos e investigadores en ciencias sociales reiteran que la satisfacción laboral es un factor determinante de la calidad de la atención, ya que la satisfacción intrínseca guarda una estrecha relación con el desempeño en el trabajo, por lo que se reconoce la vinculación causa-efecto positiva entre el clima organizacional y las actitudes favorables $\mathrm{o}$ desfavorables del trabajador (Germán Bes et al., 2011).

El trabajo es un aspecto clave en la vida de la mayoría de las personas, ya que favorece no sólo el desarrollo profesional de los individuos, sino el personal, por lo que es una de las fuentes relevantes para alcanzar metas en la vida, sin embargo, uno de los grandes retos se encuentra en el establecer el equilibrio adecuado entre el trabajo y la vida diaria, ya que dedicarle cada minuto o pensamiento puede acabar con el balance entre familia y trabajo (Parra \& Paravic, 2002). 
Lo anterior favorece que los individuos tengan la sensación de pertenencia a un sector de la sociedad y los haga sentirse valiosos e importantes, productivos. Hoy en día hay una gran preocupación por ir mejorando el entorno laboral de los trabajadores para que éstos se encuentren lo más satisfechos posible, que les haga sentir que son eficientes y que puedan enorgullecerse del trabajo que realizan (Flores García Rada, 2008). De esta manera serán capaces de adaptarse con mayor probabilidad de éxito a las exigencias del trabajo que se va incrementando a la par con el desarrollo de la humanidad.

En este estudio se visualiza que el personal de enfermería se encuentra en un nivel de satisfacción de medio a alto, donde los aspectos intrínsecos marcan la diferencia, ya que el personal se encuentra satisfecho por la labor que realiza. Evalúa como buenas y que son muy cordiales las relaciones con sus superiores y compañeros refiriendo que están de acuerdo con el 21\% (49), y el 49\% (113) respectivamente; sin embargo considera que los aspectos de promoción y competencia laboral son limitados. Con respecto a la promoción, el $51 \%$ (119) y $49 \%$ (113), están de acuerdo en tener capacidad de promoción, solo el 4\% (9) está muy en desacuerdo de estar seguro de conocer lo que se espera de su trabajo, por parte de sus jefes/as. Estas aseveraciones coinciden parcialmente con el estudio de Arias que realizo en cuatro instituciones públicas de salud del tercer nivel de atención “A, B, C, y D” en México donde menciona que el grado de satisfacción laboral e identificar los factores asociados a ésta con el personal de enfermería, satisface el sistema de trabajo y se esmeran por dar una atención de calidad al estar comprometidos con el mejoramiento del desempeño (Arias-Jiménez, 2007).

No sorprende que el concepto de satisfacción en el trabajo haya sido objeto de múltiples estudios, como lo refiere la Teoría Bifactorial de Frederick Herzberg, la cual considera los factores higiénicos del ambiente que rodea a las personas, así como las condiciones en que desempeña su trabajo. Se reconoce que la motivación laboral, internamente activa y direcciona los pensamientos y está relacionada a todos aquellos factores capaces de provocar, mantener y dirigir la conducta hacia un objetivo (De La Cruz \& Abellan, 2015; Silva, 2017), mediante los cuales los investigadores han intentado identificar las diferentes dimensiones o componentes de la satisfacción laboral, pudiendo ser predictores de aquellos factores que puedan provocar cambios intrínsecos que promuevan conductas hacia el logro de sus objetivos personales en el trabajo, así como explorar qué efecto tenían dichos componentes en la productividad de los trabajadores (Burnard, Morrison, \& Phillips, 1999), teniendo en cuenta que la satisfacción laboral está relacionada con una práctica de óptima calidad asistencial.

Definitivamente coincide el estudio que se hizo en el Hospital General de Ticomán con investigaciones realizadas, donde el personal de enfermería 
se esmera en a dar una atención de calidad, ya que se encuentra satisfecho e identificado con su profesión.

En este estudio las variables que tuvieron mayor porcentaje referidas a la satisfacción laboral fueron aquellas que se refieren a las relaciones interpersonales tanto de jefes como de compañeros. Hay que tomar en cuenta que el ser humano es único e irrepetible y sus niveles de satisfacción laboral serán también específicos, ya que están sujetos a las características personales de cada individuo y su entorno, donde juegan un papel importante aspectos tales como la historia personal, la edad, el sexo, las aptitudes, actitudes, la autoestima y el entorno sociocultural donde se desenvuelve el sujeto. Estas particularidades desarrollarán un conjunto de expectativas, necesidades y aspiraciones en relación a la vida personal y laboral (Flores García Rada, 2008; Jaramillo Antillón, 1998).

Hoy en día se ha incrementado la preocupación de los directivos por buscar una adecuada interrelación con sus empleados y el establecimiento de un buen clima organizacional, así como encontrar la manera de motivar al personal para incrementar su desempeño, interés y satisfacción en el trabajo (Flores García Rada, 2008). Representa una ventaja para las organizaciones que el trabajador realice sus actividades con gusto, que cuente con los elementos necesarios para el cumplimiento adecuado de sus responsabilidades, manteniendo y/o alcanzando la satisfacción, ya que, los trabajadores satisfechos tienden a ser más adaptables, cooperadores y dispuestos al cambio.

Las evidencias del estudio indican que los principales factores para mantener la satisfacción laboral de los profesionales enfermeros es que la parte encargada de la toma de decisiones debe propiciar un ambiente favorable, que el trabajo asignado sea intelectualmente estimulante, que se le permita al empleado desarrollar sus habilidades y la aplicación de nuevos conocimientos, así mismo que se le brinden recompensas equitativas, trato respetuoso y personalizado, lo cual aumentará el grado de satisfacción por el trabajo.

Dado el constante cambio que actualmente se vive al interior de las instituciones con respecto a los recursos, jubilaciones, contratación de personal, demanda del servicio, entre otros aspectos, se considera relevante cuidar la parte de satisfacción laboral en el personal, ya que esta repercutirá en la calidad de la atención, misma que ya presenta una crisis dadas las situaciones sociales actuales (Montes Salcedo \& Díaz Orihuela, 2020).

\section{References:}

1. Al-Shammari, M. (1992). Organizational climate. Leadership \& Organization Development Journal, 13(6), 30-32. https://doi.org/https://doi.org/10.1108/01437739210021884

2. Arias-Jiménez, M. (2007). Factores del clima organizacional 
influyentes en la satisfacción laboral de enfermería, concerniente a los cuidados intensivos neonatales del Hospital Nacional de Niños, 2004. Enfermería En Costa Rica, 28(1), 12-19. Retrieved from http://enfermeria.cr/revista/docs/Factores_del_clima_organizacional_ influyentes_en_la_satisifaccion_laboral_de_enfermeria.pdf

3. Burnard, P., Morrison, P., \& Phillips, C. (1999). Job satisfaction amongst nurses in an interim secure forensic unit in Wales. The Australian and New Zealand Journal of Mental Health Nursing, 8(1), 9-18. https://doi.org/10.1046/j.1440-0979.1999.00125.x

4. Cardona Echeverri, D. R., \& Zambrano Cruz, R. (2014). Revisión de instrumentos de evaluación de clima organizacional. Estudios Gerenciales, 30(131), 184-189. https://doi.org/10.1016/j.estger.2014.04.007

5. Carrillo-García, C., Ríos-Rísquez, M. I., Fernández-Cánovas, M. L., Celdrán-Gil, F., Vivo-Molina, M. del C., \& Martínez-Roche, M. E. (2015). La satisfacción de la vida laboral de los enfermeros de las unidades móviles de emergencias de la Región de Murcia. Enfermeria Global, 14(4), 266-275. https://doi.org/10.6018/eglobal.14.4.198161

6. Ceballos Vásquez, P. A. (2010). Desde los ámbitos de enfermería, analizando el cuidado humanizado. Ciencia y EnfermerIa, 16(1), 3135. Retrieved from https://scielo.conicyt.cl/pdf/cienf/v16n1/art_04.pdf

7. De La Cruz, S. P., \& Abellan, M. V. (2015). Desgaste profissional, stress e satisfação no trabalho do pessoal de enfermagem em um hospital universitário. Revista Latino-Americana de Enfermagem, 23(3), 543-552. https://doi.org/10.1590/0104-1169.0284.2586

8. Figueiredo Manzo, B., Teodoro Couto Ribeiro, H. C., Menezes Brito, M. J., \& Alves, M. (2012). La enfermería en hospital en proceso de acreditación: su papel y las consecuencias en el trabajo diario. Rev. Latino-Am.Enfermagem, 20(1), 1-8. Retrieved from http://www.scielo.br/pdf/rlae/v20n1/es_20.pdf

9. Flores García Rada, J. (2008). El comportamiento humano en las organizaciones. (U. del Pacífico, Ed.). Lima.

10. Germán Bes, C., Hueso Navarro, F., \& Huércanos Esparza, I. (2011). El cuidado en peligro en la sociedad global. Enfermería Global, 10(23), 221-232. https://doi.org/10.4321/s1695-61412011000300016

11. Hernández Zavala, M., Hernández Cantoral, A., Nava Galán, M. G., Pérez López, M. T., Hernández Ramírez, M. G., Matus Miranda, R., \& Balseiro Almario, C. L. (2012). Satisfacción laboral del profesional de enfermería en cuatro instituciones de salud. Enfermería Uni, 9(1), 7-15. Retrieved from http://www.scielo.org.mx/scielo.php?script=sci_arttext\&pid=S1665- 
70632012000100002

12. Herrera-Amaya, G., \& Manrique-Abril, F. (2019). Satisfacción laboral de enfermería. Diferencias de ámbitos en atención primaria en salud y cuidado intensivo. Duazary, 16(2), 74-86. https://doi.org/10.21676/2389783X.2943

13. Jaramillo Antillón, J. (1998). Principios de gerencia y administración de servicios médicos y hospitales. (U. de C. Rica, Ed.). Costa Rica: Editorial Nacional de Salud y Seguridad Social.

14. Molina Linde, J. M., Avalos Martínez, F., Valderrama Orbegozo, L. J., \& Uribe Rodríguez, A. F. (2009). Factores relacionados con la satisfacción laboral de enfermería en un hospital médico-quirúrgico. Investigación y Educación En Enfermería, 27(2), 218-225. Retrieved from http://www.scielo.org.co/pdf/iee/v27n2/v27n2a07.pdf

15. Montes Salcedo, M., \& Díaz Orihuela, M. M. (2020). Motivación y satisfacción laboral del personal de Enfermería en el Hospital de Apoyo San Francisco, Ayacucho-2018. Revista Científica de Ciencias de La Salud, 12(2), 0-3. https://doi.org/10.17162/rccs.v12i2.892

16. Núñez González, E., Estévez Guerra, G. J., Hernández Marrero, P., \& Marrero Medina, C. D. (2007). Una propuesta destinada a complementar el cuestionario Font-Roja de satisfacción laboral. Gaceta Sanitaria, 21(2), 136-141. https://doi.org/10.1157/13101040

17. Parra, S., \& Paravic, T. (2002). Satisfacción laboral en enfermeras/os que trabajan en el sistema de atención médica de urgencia (SAMU). Ciencia y Enfermeria, 8(2), 37-48.

https://doi.org/http://dx.doi.org/10.4067/S0717-95532002000200005

18. Piqueras Gómez, R., Rodríguez Morejón, A., \& Rueda Sabater, C. (2008). Expectativas y duración del desempleo. Revista de Psicología Del Trabajo y de Las Organizaciones, 24(2), 129-151. Retrieved from https://www.redalyc.org/articulo.oa?id=231316495001

19. Ponce-Gómez, J., Ponce-Gómez, G., \& Lugo-Chávez, I. (2009). Satisfacción laboral del personal de enfermería en un hospital de gineco-obstetricia del Instituto Mexicano del Seguro Social. Rev Enferm Inst Mex Seguro, 17(18), 103-108. Retrieved from https://www.medigraphic.com/pdfs/enfermeriaims s/eim2009/eim092h.pdf

20. Sánchez Jacas, I., Brea López, I., De La Cruz Castro, M., \& Matos Fernández, I. (2017). Motivación y liderazgo del personal del subsistema de servicios generales en dos hospitales maternos. Correo Científico Médico, 21(2), 434-444. Retrieved from http://revcocmed.sld.cu/index.php/cocmed/article/view/2284/1040

21. Silva, M. C. (2017). Sufrimiento laboral en el equipo de un centro de salud familiar rural del sur de Chile. Rev Chil Salud Pública, 21(1), 
10-18. Retrieved from

https://revistasaludpublica.uchile.cl/index.php/RCSP/article/view/476 54

22. Soto, P. E., Barrios, S., \& Molina, Y. (2017). Síndrome de quemarse por el trabajo y satisfacción laboral como predictores de calidad de la atención de Enfermería Hospitalaria. Ciencia y Enfermería, 23(3), 99111. https://doi.org/10.4067/s0717-95532017000300099

23. Troya, C., Padilla, M., Camacho, L., \& BenavidesLaura. (2020). Evaluación de la satisfacción laboral en un grupo de enfermeras de cuatro ciudades del Ecuador en el mes de enero de 2020. Practica Familiar Rural, 5(1).

https://doi.org/https://doi.org/10.23936/pfr.v5i1.145 